\title{
Correlação de variáveis espectrais e estoque de carbono da biomassa aérea de sistemas agroflorestais
}

\author{
Édson Luis Bolfe ${ }^{(1)}$, Mateus Batistella(1) e Marcos César Ferreira ${ }^{(2)}$ \\ (1)Embrapa Monitoramento por Satélite, Avenida Soldado Passarinho, no 303, CEP 13070-115 Campinas, SP. E-mail: bolfe@cnpm.embrapa.br, \\ mb@cnpm.embrapa.br (2)Universidade Estadual de Campinas, Rua João Pandiá Calógeras, no 51, CEP 13083-870 Campinas, SP. \\ E-mail: macferre@ige.unicamp.br
}

\begin{abstract}
Resumo - O objetivo deste trabalho foi avaliar a correlação entre variáveis espectrais e o estoque de carbono da biomassa aérea de sistemas agroflorestais da região de Tomé-Açu, PA. Foram testados 24 índices de vegetação de três grupos (razão simples, diferença normalizada e complexos), gerados a partir de imagens do sensor TM/ Landsat-5, adquiridas em 2008. As variáveis obtidas foram correlacionadas, por meio de regressão linear simples, ao estoque de carbono de quatro classes de sistemas agroflorestais, de diferentes idades e composições florísticas. As correlações obtidas entre as variáveis espectrais e o estoque de carbono foram significativas em $47 \%$ dos índices testados e variaram de acordo com as diferenças de biomassa nos sistemas analisados. As melhores correlações foram obtidas pelos índices de vegetação de razão simples e de diferença normalizada, em sistemas agroflorestais jovens, e pelos índices de vegetação complexos, em sistemas agroflorestais mais antigos.
\end{abstract}

Termos para indexação: integração lavoura-pecuária-floresta, Landsat, sensoriamento remoto, uso e cobertura da terra.

\section{Correlation of spectral variables and aboveground carbon stock of agroforestry systems}

\begin{abstract}
The objective of this work was to evaluate the correlation between spectral variables and aboveground carbon stock of agroforestry systems in the region of Tomé-Açu, PA, Brazil. Twenty-four vegetation indices from three groups (simple ratio, normalized difference, and complex), calculated from images of the TM/ Landsat- 5 sensor acquired in 2008, were tested. The obtained variables were correlated, by means of simple linear regression, to carbon stock from four agroforestry systems with different ages and floristic composition. The correlations obtained among spectral variables and carbon stock were significant in $47 \%$ of the tested indices and changed according to the differences in biomass of the analyzed systems. The best correlations were obtained by the simple ratio and normalized difference indices in young agroforestry systems, and by complex vegetation indices in older agroforestry systems.
\end{abstract}

Index terms: crop-livestock-forest integration, Landsat, remote sensing, land use and land cover.

\section{Introdução}

A cobertura florestal da Amazônia tem sofrido mudanças significativas na última década (Instituto Nacional de Pesquisas Espaciais, 2008). Essas alterações do uso e da cobertura da terra estão relacionadas, principalmente, ao desmatamento de sistemas florestais para transformação em sistemas agrícolas ou pastagem, o que resulta em transferência de carbono da biosfera para a atmosfera e contribui para alterações climáticas de escala local, regional e global (Nobre et al., 2007).

Os sistemas agroflorestais (SAF) são importantes componentes do mosaico de uso e cobertura da terra existente na Amazônia, particularmente por sua contribuição com serviços ecossistêmicos. Ao analisar as condições de agroflorestas em Tomé-Açu, PA, Pinho et al. (2012) observaram que estes sistemas - que combinam cultivos diversificados - apresentam características ambientais favoráveis ao acúmulo de biomassa na vegetação. Esta condição tem efeito positivo sobre matéria orgânica, infiltração de água e fixação de carbono no solo e na vegetação.

A estimativa de biomassa de sistemas florestais permite amparar decisões vinculadas a atividades econômicas e ambientais, como políticas públicas, manejo florestal, estudos de ciclagem de nutrientes e ordenamento territorial (Lu et al., 2005). De acordo com Tsukamoto et al. (2004), os estudos de estimativa de

Pesq. agropec. bras., Brasília, v.47, n.9, p.1261-1269, set. 2012 
carbono em agroflorestas baseiam-se, exclusivamente, em métodos destrutivos. Esses métodos apresentam etapas laboriosas na amostragem da biomassa aérea, e têm resultados restritos aos pontos de coleta de dados.

Métodos baseados em características locais, mas passíveis de extrapolação para a paisagem analisada, são mais eficazes em estimar o carbono da biomassa e possuem diversas aplicações. Trabalhos de quantificação indireta com enfoque em processos não destrutivos, como os que usam correlações entre variáveis espectrais e de campo, ainda estão em fase inicial de desenvolvimento e constituem desafio técnico-científico para a melhor compreensão dos fluxos de carbono (Lu et al., 2005).

Índices de vegetação são algoritmos baseados na transformação de valores de refletância de determinadas imagens, calculados pormeio de diferentes combinações lineares de respostas espectrais. Segundo Rizzi \& Rudorff (2007), dados obtidos por meio de variáveis espectrais servem como indicadores do crescimento e do vigor de vegetação verde e podem ser utilizados no diagnóstico de vários parâmetros biofísicos, como a biomassa aérea. As variáveis espectrais advindas de índice de vegetação são derivadas, basicamente, de imagens de satélite na região do vermelho $(0,65$ a $0,72 \mu \mathrm{m})$ e do infravermelho próximo $(0,7$ e $1,3 \mu \mathrm{m})$, no espectro eletromagnético, e são frequentemente empregadas na estimativa não destrutiva da biomassa (Liu \& Kogan, 2002). No entanto, as extrapolações das relações entre índices de vegetação e valores de biomassa determinados no campo, para comunidades vegetais, apresentam dificuldades não totalmente resolvidas (Foody et al., 2003).

O objetivo deste trabalho foi avaliar a correlação entre variáveis espectrais e o estoque de carbono da biomassa aérea de sistemas agroflorestais da região de Tomé-Açu, PA.

\section{Material e Métodos}

O trabalho foi realizado em áreas de produtores rurais vinculados à Cooperativa Agrícola Mista de Tomé-Açu (Camta), localizada no distrito de Quatro Bocas, Município de Tomé-Açu, PA. A agricultura é a base da economia local, com destaque para a produção de pimenta-do-reino e a fruticultura tropical de espécies nativas e exóticas, desenvolvida em agroflorestas.
O relevo é caracterizado por baixos platôs aplainados (tabuleiros), terraços e várzeas, entre 14 e $96 \mathrm{~m}$ de altitude. Ocorrem solos classificados como Latossolo Amarelo distrófico (LAd), com textura argilosa média, Plintossolos (F), Gleissolos (G), e Neossolos Flúvicos eutróficos (RYe) e distróficos (RYd), em associação (Rodrigues et al., 2001). A vegetação é composta por Floresta Ombrófila Densa de Terras Baixas, atualmente bastante alterada.

O clima, de acordo com a classificação de Köppen, é do tipo Ami, mesotérmico e úmido, com temperatura média anual de $26^{\circ} \mathrm{C}$ e umidade relativa do ar em torno de $85 \%$. A média anual de precipitação é de $2.300 \mathrm{~mm}$, com média mensal entre $54 \mathrm{~mm}$ em agosto, e $440 \mathrm{~mm}$ em março. No trimestre mais chuvoso (fevereiro, março e abril), as médias mensais ultrapassam $300 \mathrm{~mm}$, e, no menos chuvoso (agosto, setembro e outubro), ficam próximas de $60 \mathrm{~mm}$ (Pachêco \& Bastos, 2008).

Foram identificados 217 lotes de agricultores com potencial para os levantamentos de campo na região (sócios e não sócios da Camta). Destes, foram selecionados 40 lotes de forma aleatória. As parcelas foram constituídas por três unidades amostrais de $900 \mathrm{~m}^{2}$ cada uma, o que totalizou 120 unidades (Figura 1). As coordenadas geográficas do centro de cada amostra foram obtidas por meio de sistema de posicionamento global (GPS), para posterior identificação nas imagens de satélites.

A partir da análise da estrutura da vegetação, foram definidas quatro classes de sistemas agroflorestais (SAF 1, SAF 2, SAF 3 e SAF 4), de acordo com a altura total $(\mathrm{H})$ e o diâmetro à altura do peito (DAP) dos indivíduos (Bolfe \& Batistella, 2011). Esses sistemas apresentavam diferentes idades e composições florísticas, com biomassa e cobertura da terra crescente do SAF 1 para o SAF 4. Os dados foram utilizados em modelos alométricos desenvolvidos para a região amazônica (Higuchi et al., 1998; Bartelt et al., 2000), para calcular a biomassa epígea seca e estimar o carbono em cada unidade amostral, conforme Bolfe et al. (2009). No trabalho de campo, realizado em agosto de 2008, 57 parcelas foram inventariadas nos lotes identificados inicialmente. Determinou-se a biomassa área por parcela, para validação dos dados.

As variáveis espectrais foram obtidas a partir de imagem do sensor TM/Landsat-5, de 13/6/2008 (período vegetativo da missão de campo), órbita/ponto 223/062. As imagens utilizadas foram processadas digitalmente 


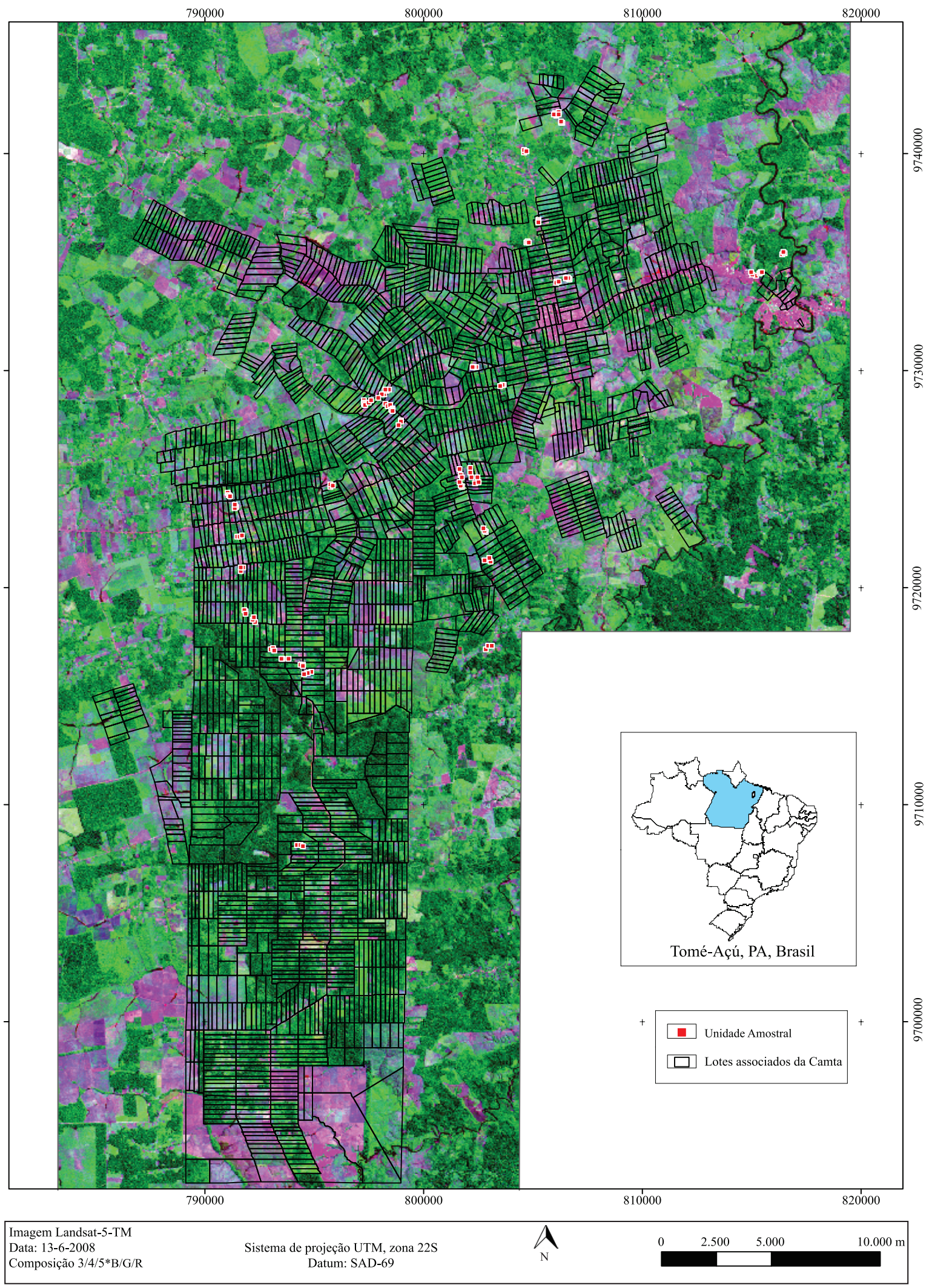

Figura 1. Imagem do sensor TM/Landsat-5, com a área de estudo: lotes de agricultores e unidades amostrais na Região de Tomé-Açu, PA, em 2008. 
para minimizar alterações detectadas, o que inclui a atenuação de distorções geométricas e radiométricas. Esta técnica modifica os números digitais (ND) para caracterizar as imagens no caso de recepção ideal, o que engloba as distorções provocadas pela atmosfera e a conversão de ND da imagem em parâmetros físicos (radiância e reflectância). Em razão da dificuldade de se obter parâmetros atmosféricos fidedignos da região amazônica, para utilização em modelos complexos de correção atmosférica, conforme recomendado por $\mathrm{Lu}$ et al. (2002), utilizou-se o modelo de subtração de objeto escuro (DOS), mais simples. Neste processo, foram empregados coeficientes de calibração do sensor TM e demais parâmetros, conforme National Aeronautics and Space Administration (2008). Posteriormente, as imagens foram retificadas geometricamente para a projeção "universal transversa de mercator" (UTM) (Zona 22 S, SAD 69). A reamostragem foi efetuada por meio do interpolador "vizinho mais próximo", e o erro médio quadrático situou-se em torno de 0,5 pixel.

Ao se considerar as características dos sistemas florestais avaliados (biodiversidade, idade, cobertura vegetal e exposição do solo), foram testados 24 índices de vegetação, subdivididos em três grupos: índices de razão simples, índices normalizados e índices complexos (ortogonais ou perpendiculares) (Tabela 1). Os índices de razão simples e normalizados baseiamse no contraste entre a resposta da vegetação na faixa do vermelho e do infravermelho próximo, médio e distante. Já os índices complexos, consideram o contraste espectral existente entre a vegetação e as características do solo. Os solos expostos em um gráfico bidimensional formado por uma banda no visível versus uma banda no infravermelho próximo ocorrem, aproximadamente, em uma linha, denominada "linha do solo" (Demattê \& Nanni, 2003).

Tabela 1. Índices de vegetação utilizados na obtenção das variáveis espectrais dos sistemas agroflorestais avaliados em Tomé-Açu, PA.

\begin{tabular}{|c|c|c|}
\hline Equação & Indice ${ }^{(1)}$ & Referências \\
\hline \multicolumn{3}{|c|}{ Razão simples } \\
\hline$\left(\rho_{\mathrm{IVP}} / \rho_{\mathrm{V}}\right)$ & RVI 4:3 & Jordan (1969) \\
\hline$\left(\rho_{\mathrm{V}} / \rho_{\mathrm{IVP}}\right)$ & RVI $3: 4$ & Jordan (1969) \\
\hline$\left(\rho_{\mathrm{IVM}} / \rho_{\mathrm{V}}\right)$ & RVI 5:3 & Jordan (1969) \\
\hline$\left(\rho_{\mathrm{IVM}} / \rho_{\mathrm{IVP}}\right)$ & RVI 5:4 & Jordan (1969) \\
\hline \multicolumn{3}{|c|}{ Normalizados } \\
\hline$\left(\rho_{\mathrm{IVP}}-\rho_{\mathrm{V}}\right) /\left(\rho_{\mathrm{IVP}}+\rho_{\mathrm{V}}\right)$ & NDVI $4: 3$ & Huete (2002) \\
\hline$\left(\rho_{\mathrm{IVM}}-\rho_{\mathrm{V}}\right) /\left(\rho_{\mathrm{IVM}}+\rho_{\mathrm{V}}\right)$ & NDVI 5:3 & Huete (2002) \\
\hline$\left(\rho_{\mathrm{IVM}}-\rho_{\mathrm{IVP}}\right) /\left(\rho_{\mathrm{IVM}}+\rho_{\mathrm{IVP}}\right)$ & NDVI 5:4 & Huete (2002) \\
\hline$\left(\rho_{\mathrm{IVM}}-\rho_{\mathrm{IVD}}\right) /\left(\rho_{\mathrm{IVM}}+\rho_{\mathrm{IVD}}\right)$ & NDVI 5:7 & Huete (2002) \\
\hline$\sqrt{ }\left(\left(\rho_{\mathrm{IVP}}-\rho_{\mathrm{V}}\right) /\left(\rho_{\mathrm{IVP}}+\rho_{\mathrm{V}}\right)\right)+0,5$ & TVI & Rouse et al. (1974) \\
\hline$\left(\left(\rho_{\mathrm{IVP}}-\rho_{\mathrm{V}}\right) /\left(\rho_{\mathrm{IVP}}+\rho_{\mathrm{V}}\right)\right)+0,5$ & \multirow{2}{*}{ CTVI } & \multirow{2}{*}{ Perry \& Lautenschlager (1984) } \\
\hline$\left|\left(\left(\rho_{\mathrm{IVP}}-\rho_{\mathrm{V}}\right) /\left(\rho_{\mathrm{IVP}}+\rho_{\mathrm{VV}}\right)\right)+0,5\right| \times \sqrt{ } \mid\left(\left(\rho_{\mathrm{IVP}}-\rho_{\mathrm{V}}\right) /\left(\rho_{\mathrm{IVP}}+\rho_{\mathrm{V}}\right.\right.$ & & \\
\hline$\sqrt{ }\left|\left(\left(\rho_{\mathrm{IVP}}-\rho_{\mathrm{V}}\right) /\left(\rho_{\mathrm{IVP}}+\rho_{\mathrm{V}}\right)\right)+0,5\right|$ & TTVI & Silleos et al. (2006) \\
\hline$\left(\left(\rho_{\mathrm{VP}} / \rho_{\mathrm{V}}\right)-1\right) /\left(\left(\rho_{\mathrm{IVP}} / \rho_{\mathrm{V}}\right)+1\right)$ & NRVI & Baret \& Guyot (1991) \\
\hline \multicolumn{3}{|c|}{ Complexos (ortogonais ou perpendiculares) } \\
\hline$\sqrt{ }(\operatorname{Rgg} 5-\operatorname{Rp} 5)^{2}+(\operatorname{Rgg} 7-\operatorname{Rp} 7)^{2}$ & PVI & Richardson \& Wiegand (1977) \\
\hline$\left(b \rho_{\mathrm{IVP}}-\rho_{\mathrm{V}}+\mathrm{a}\right) /\left(\sqrt{ }\left(\mathrm{b}^{2}+1\right)\right)$ & PVI 1 & Perry \& Lautenschlager (1984) \\
\hline$\left[\left(\rho_{\mathrm{IVP}}-\mathrm{a}\right) \times\left(\rho_{\mathrm{V}}+\mathrm{b}\right)\right] /\left(\sqrt{ }\left(1+\mathrm{a}^{2}\right)\right)$ & PVI 2 & Perry \& Lautenschlager (1984) \\
\hline$\left(a \rho_{I V P}-b \rho_{V}\right)$ & PVI 3 & Perry \& Lautenschlager (1984) \\
\hline$\left[\left(\rho_{\mathrm{IVP}}-\rho_{\mathrm{V}}\right) /\left(\rho_{\mathrm{IVP}}+\rho_{\mathrm{V}}\right)\right] \times(1+\mathrm{L})$ & SAVIa,b,c & Huete (1988) \\
\hline $\mathrm{a}\left(\rho_{\mathrm{IVP}}-\mathrm{a} \times \rho_{\mathrm{V}}-\mathrm{b}\right) /\left(\rho_{\mathrm{V}}+\mathrm{a} \times \rho_{\mathrm{IVP}}-\mathrm{a} \times \mathrm{b}\right)$ & TSAVI 1 & Baret \& Guyot (1991) \\
\hline $\mathrm{a}\left(\rho_{\mathrm{IVP}}-\mathrm{a} \times \rho_{\mathrm{V}}-\mathrm{b}\right) /\left[\rho_{\mathrm{V}}+\mathrm{a} \times \rho_{\mathrm{IVP}}-\mathrm{a} \times \mathrm{b}+0,8\left(1+\mathrm{a}^{2}\right)\right]$ & TSAVI 2 & Baret \& Guyot (1991) \\
\hline$\left[\left(2 \rho_{\mathrm{IVP}}+1\right)-\sqrt{ }\left(2 \rho_{\mathrm{IVP}}+1\right)^{2}-8\left(\rho_{\mathrm{IVP}}-\rho_{\mathrm{V}}\right)\right] / 2$ & MSAVI 2 & Qi et al. (1994) \\
\hline $\mathrm{a}\left(\rho_{\mathrm{IVP}}-\rho_{\mathrm{V}}\right)$ & DVI & Richardson \& Wiegand (1977) \\
\hline$\left(\rho_{\mathrm{IVP}}-\mathrm{c} \times \rho_{\mathrm{V}}\right)$ & WDVI & Clevers (1988) \\
\hline
\end{tabular}

${ }^{(1)}$ RVI, "ratio vegetation index"; NDVI, índice de vegetação por diferença normalizada; TVI, "transformation vegetation index"; CTVI, "corrected transformed vegetation index; TTVI, "Thiam's transformed vegetation index"; NRVI, "normalized ratio vegetation index"; PVI (1, 2, 3), "perpendicular vegetation index"; SAVI, "soil adjusted vegetation index"; TSAVI $(1,2)$, "transformed soil adjusted vegetation index"; MSAVI $(1,2)$, "modified soil adjusted vegetation index"; DVI, "difference vegetation index"; WDVI, "weighted difference vegetation index". 
A correlação entre os parâmetros obtidos em campo, nas diferentes classes de SAF, e as variáveis espectrais, nos diferentes grupos, foi realizada em sistema de informações geográficas (SIG). Esta rotina foi gerada da função "extract", em que foram obtidos os valores do estoque de carbono nos pontos amostrais $\left(\mathrm{Mg} \mathrm{ha}^{-1}\right)$ e os valores das médias espectrais (ND) dos índices de vegetação nesses pontos. Em seguida, aplicou-se a análise de regressão linear simples, em que a variável independente foi o valor do número digital de cada índice de vegetação testado, e a variável dependente, o valor para o conteúdo de carbono em cada sistema agroflorestal.

Como última etapa, diferentes modelos foram analisados a partir dos coeficientes $\mathrm{r}$ e $\mathrm{R}^{2}$, pelo teste $\mathrm{t}$ de Student, a 1 e $5 \%$ de probabilidade.

\section{Resultados e Discussão}

A composição florística das unidades amostrais apresentou 54 espécies de 27 famílias botânicas, com diferentes indivíduos arbóreos e arbustivos: Theobroma cacao L. (cacau), Euterpe oleracea Mart. (açaí), Theobroma grandiflorum (Willd. Ex Spreng.) K.Schum (cupuaçu) e Hevea brasiliensis (Willd. ex A. Juss.) Müll. Arg. (seringueira). Essas espécies representaram, conjuntamente, $51 \%$ da frequência relativa, $69,2 \%$ da densidade relativa e $51,1 \%$ da dominância relativa. Entre as espécies menos frequentes, foram observadas: Annona muricata L. (Graviola), Gliricidia sp. (gliricídia), Bertholletia excelsa Bonpl. (castanheira), Carapa guianensis Aubl. e Musa paradisiaca L. (bananeira).

As médias \pm erro-padrão de carbono nos sistemas agroflorestais foram: SAF 1, 2,83 $\pm 1,13 \mathrm{Mg} \mathrm{ha}^{-1}$; SAF 2, $18,85 \pm 6,22 \mathrm{Mg} \mathrm{ha}^{-1}$; SAF 3, 36,58 $\pm 5,58 \mathrm{Mg} \mathrm{ha}^{-1}$; e SAF 4, 114,58 $\pm 34,05 \mathrm{Mg} \mathrm{ha}^{-1}$. A média geral do estoque de carbono das classes $\left(43,21 \mathrm{Mg} \mathrm{ha}^{-1}\right)$ é próxima da média estimada por Montagnini \& Nair (2004), ao avaliar a vegetação sistemas agroflorestais na Amazônia (50 $\left.\mathrm{Mg} \mathrm{ha}^{-1}\right)$.

A partir dos valores dos 24 índices de vegetação, divididos por grupo, foram geradas 96 correlações, das quais 45 foram significativas (Tabela 2). Isso mostra a diversidade expressada pelos índices e suas diferentes relações com a quantidade de carbono medida em campo.

Os resultados observados para as classes de sistemas agroflorestais avaliadas foram: SAF $1\left(\mathrm{R}^{2}=0,54\right.$ no "ratio vegetation index" - RVI 5:3), SAF $2\left(\mathrm{R}^{2}=0,73\right.$ no índice de vegetação por diferença normalizada - NDVI 5:7), SAF $3\left(\mathrm{R}^{2}=0,78\right.$ no "transformed soil adjusted vegetation index" - TSAVI 2) e SAF $4\left(\mathrm{R}^{2}=0,61\right.$ no "perpendicular vegetation index" - PVI 3) (Tabela 2). Esses valores são superiores aos obtidos por Foody et al. (2003), que relacionaram a biomassa de florestas tropicais no Estado do Amazonas com 11 índices de vegetação gerados por meio de imagens $\mathrm{TM} /$ Landsat-5 $\left(\mathrm{R}^{2}=0,50\right)$, e por Accioly et al. (2002), que estimaram parâmetros estruturais de vegetação de Floresta Tropical e de Cerrado, obtidos via índices de vegetação e modelo

Tabela 2. Estatísticas das correlações e análises de regressão entre classes de sistemas agroflorestais e índices de vegetação.

\begin{tabular}{|c|c|c|c|c|c|c|c|c|}
\hline \multirow[t]{2}{*}{ Índices } & \multicolumn{2}{|c|}{ SAF 1} & \multicolumn{2}{|c|}{ SAF 2} & \multicolumn{2}{|c|}{ SAF 3} & \multicolumn{2}{|c|}{ SAF 4} \\
\hline & $r$ & $\mathrm{R}^{2}$ & $\mathrm{r}$ & $\mathrm{R}^{2}$ & $\mathrm{r}$ & $\mathrm{R}^{2}$ & $\mathrm{r}$ & $\mathrm{R}^{2}$ \\
\hline & \multicolumn{8}{|c|}{ Razão simples } \\
\hline RVI & $-0,61$ & $0,37^{\mathrm{ns}}$ & 0,78 & $0,62 * *$ & $-0,80$ & $0,65^{* *}$ & 0,23 & $0,05^{\mathrm{ns}}$ \\
\hline RVI 3:4 & 0,57 & $0,32^{\mathrm{ns}}$ & $-0,80$ & $0,65^{* *}$ & 0,79 & $0,63 * *$ & $-0,38$ & $0,14^{\mathrm{ns}}$ \\
\hline RVI 5:3 & $-0,73$ & $0,54^{*}$ & 0,18 & $0,03^{\mathrm{ns}}$ & $-0,29$ & $0,08^{\mathrm{ns}}$ & 0,51 & $0,26^{\mathrm{ns}}$ \\
\hline \multirow[t]{2}{*}{ RVI 5:4 } & 0,43 & $0,19^{\text {ns }}$ & $-0,83$ & $0,70 * *$ & 0,69 & $0,48^{*}$ & $-0,01$ & $0,03^{\mathrm{ns}}$ \\
\hline & \multicolumn{8}{|c|}{ Complexos } \\
\hline NDVI4:3 & 0,60 & $0,36^{\text {ns }}$ & $-0,80$ & $0,64 * *$ & 0,81 & $0,66^{* *}$ & $-0,28$ & $0,08^{\mathrm{ns}}$ \\
\hline NDVI5:3 & $-0,73$ & $0,53^{*}$ & 0,14 & $0,02^{\mathrm{ns}}$ & $-0,27$ & $0,07^{\mathrm{ns}}$ & 0,52 & $0,27 *$ \\
\hline NDVI 5:4 & 0,48 & $0,23^{\text {ns }}$ & $-0,84$ & $0,71 * *$ & 0,73 & $0,53 * *$ & 0,02 & $0,08^{\mathrm{ns}}$ \\
\hline NDVI 5:7 & $-0,48$ & $0,23^{\text {ns }}$ & 0,85 & $0,73 * *$ & $-0,69$ & $0,48 * *$ & 0,36 & $0,13^{\mathrm{ns}}$ \\
\hline TVI & 0,61 & $0,37^{\text {ns }}$ & $-0,80$ & $0,64 * *$ & 0,81 & $0,65 * *$ & $-0,26$ & $0,07^{\mathrm{ns}}$ \\
\hline CTVI & 0,61 & $0,37^{\text {ns }}$ & $-0,80$ & $0,64 * *$ & 0,81 & $0,65^{* *}$ & $-0,26$ & $0,07^{\mathrm{ns}}$ \\
\hline TTVI & 0,61 & $0,37^{\text {ns }}$ & $-0,80$ & $0,64 * *$ & 0,81 & $0,65^{* *}$ & $-0,27$ & $0,07^{\mathrm{ns}}$ \\
\hline \multirow[t]{2}{*}{ NRVI } & $-0,60$ & $0,36^{\text {ns }}$ & 0,80 & $0,64 * *$ & $-0,81$ & $0,66 * *$ & 0,28 & $0,08^{\mathrm{ns}}$ \\
\hline & \multicolumn{8}{|c|}{ Normalizados } \\
\hline PVI & $-0,60$ & $0,36^{\text {ns }}$ & 0,62 & $0,39 *$ & $-0,34$ & $0,12^{\mathrm{ns}}$ & 0,70 & $0,49 * *$ \\
\hline PVI 1 & 0,60 & $0,36^{\text {ns }}$ & $-0,62$ & $0,39 *$ & 0,34 & $0,12^{\mathrm{ns}}$ & 0,70 & $0,49 * *$ \\
\hline PVI 2 & 0,60 & $0,36^{\text {ns }}$ & $-0,56$ & $0,32^{\mathrm{ns}}$ & 0,26 & $0,07^{\text {ns }}$ & $-0,73$ & $0,54^{* *}$ \\
\hline PVI 3 & 0,55 & $0,31^{\text {ns }}$ & $-0,36$ & $0,13^{\mathrm{ns}}$ & 0,08 & $0,07^{\text {ns }}$ & $-0,78$ & $0,61^{* *}$ \\
\hline SAVIa & 0,61 & $0,37^{\text {ns }}$ & $-0,76$ & $0,58^{* *}$ & 0,69 & $0,47^{*}$ & $-0,49$ & $0,24 *$ \\
\hline SAVIb & 0,61 & $0,37^{\text {ns }}$ & $-0,74$ & $0,54 * *$ & 0,61 & $0,37^{\text {1s }}$ & $-0,57$ & $0,32 *$ \\
\hline SAVIc & 0,61 & $0,37^{\text {ns }}$ & $-0,71$ & $0,50^{*}$ & 0,53 & $0,28^{\text {ns }}$ & $-0,62$ & $0,39^{* *}$ \\
\hline TSAVI 1 & $-0,58$ & $0,33^{\text {ns }}$ & $-0,01$ & $0,02^{\text {ns }}$ & $-0,29$ & $0,08^{\mathrm{ns}}$ & $-0,70$ & $0,50^{* *}$ \\
\hline TSAVI 2 & 0,60 & $0,36^{\mathrm{ns}}$ & $-0,79$ & $0,63^{* *}$ & 0,88 & $0,78^{* *}$ & 0,10 & $0,01^{\text {ns }}$ \\
\hline MSAVI 2 & 0,61 & $0,37^{\text {ns }}$ & $-0,74$ & $0,55^{* *}$ & 0,63 & $0,40^{*}$ & $-0,57$ & $0,33^{*}$ \\
\hline DVI & 0,60 & $0,36^{\text {ns }}$ & $-0,62$ & $0,39^{*}$ & 0,34 & $0,12^{\text {ns }}$ & $-0,70$ & $0,49^{* *}$ \\
\hline WDVI & 0,60 & $0,36^{\text {ns }}$ & $-0,57$ & $0,33^{\mathrm{ns}}$ & 0,27 & $0,07^{\text {ns }}$ & $-0,73$ & $0,53^{* *}$ \\
\hline
\end{tabular}

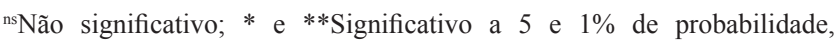
respectivamente. RVI, "ratio vegetation index"; NDVI, índice de vegetação por diferença normalizada; SAVI, "soil adjusted vegetation index"; TVI, "transformation vegetation index"; CTVI, "corrected transformed vegetation index"; TTVI, "Thiam's transformed vegetation index"; NRVI, "normalized ratio vegetation index"; PVI $(1,2,3)$, "perpendicular vegetation index"; TSAVI $(1,2)$, "transformed soil adjusted vegetation index"; MSAVI $(1,2)$, "modified soil adjusted vegetation index"; DVI, "difference vegetation index"; WDVI, "weighted difference vegetation index". 
linear de mistura espectral, em imagens TM/Landsat-5 $\left(\mathrm{R}^{2}=0,33\right.$ a 0,60$)$. Os valores obtidos para a região de Tomé-Açu também foram superiores aos observados por Lu et al. (2005), na correlação entre biomassa aérea e índices de vegetação, texturas e modelo de mistura espectral para florestas tropicais primárias $\left(\mathrm{R}^{2}=0,12\right.$ a $0,18)$. Entretanto, foram semelhantes aos relatados por Ponzoni \& Rezende (2004) $\left(\mathrm{R}^{2}=0,50\right.$ a 0,76$)$ e por $\mathrm{Lu}$ et al. (2005) $\left(\mathrm{R}^{2}=0,69\right.$ a 0,81$)$, para florestas tropicais secundárias, com uso de imagens TM/Landsat-5.

Nas áreas amostrais classificadas como SAF 1, representadas pela imagem de razão simples RVI 5:3, os menores valores indicam maior densidade de cobertura vegetal e os valores mais elevados representam solo nu ou sem vegetação (Figura 2 A). Assim, os valores observados, entre 0,35 e 0,40 , são decorrentes da baixa cobertura vegetal dessa classe de agrofloresta, na qual o recobrimento de copa era inferior a $30 \%$ e o estoque de carbono situou-se em torno de $6 \mathrm{Mg} \mathrm{ha}^{-1}$.

A cobertura vegetal da área em destaque, na imagem síntese do índice de razão simples RVI 5:3 (Figura 2 B), é composta, principalmente, por gramíneas e indivíduos arbóreos em fase inicial de desenvolvimento (até dois anos), onde o solo exposto exerce influência. Os índices de vegetação mais sensíveis à influência do solo exposto, como o NDVI e os derivados do TSAVI, não apresentaram correlações significativas com a biomassa. No entanto, os índices que tiveram em sua composição as bandas 3 e 5 foram os que apresentaram melhores correlações $\left(\mathrm{R}^{2}=0,54\right.$ no índice RVI $5: 3$ e $\mathrm{R}^{2}=0,53$ no índice NDVI 5:3) (Tabela 2). A aplicação da banda 5 do TM/Landsat-5 é recomendada por Lu et al. (2005) e Freitas et al. (2005) para correlações com a biomassa, principalmente em áreas florestais estabelecidas, já que esta banda espectral, em separado, apresenta forte influência da estrutura de dossel. Ao subtrair a banda 3 (absorção da borda do vermelho) nestes índices, destaca-se o solo e retiram-se as características vegetacionais, o que torna a correlação negativa, mas satisfatória. Isso indica baixa correlação para esta classe (SAF 1), composta, essencialmente, por vegetação esparsa e com influência do solo. Contudo, os índices mais preparados para atenuar a influência de solo não apresentaram melhores resultados em razão da diversidade dos componentes vegetacionais (gramíneos e arbóreos), que dificultou a calibração pelos fatores de ajuste de solo e fez com que os valores de biomassa não fossem elevados o suficiente para uma diferenciação satisfatória.
Na classe SAF 2, a maior correlação foi observada no grupo dos índices normalizados (NDVI 5:7), com $\mathrm{R}^{2}=0,73$. A imagem NDVI 5:7 na Figura $2 \mathrm{C}$ destacou a área amostral do SAF 2, e representou cobertura vegetal dessa classificação agroflorestal. Embora os indivíduos arbóreos apresentem, nessa classe, altura superior a $3 \mathrm{~m}$ e idade mais elevada (até cinco anos), o recobrimento de copa (45\%) e a média de carbono (22 $\mathrm{Mg} \mathrm{ha}^{-1}$ ) ainda são baixos, o que faz com que a área seja considerada como de agrofloresta relativamente jovem. Observou-se o início da divisão de dois estratos vegetativos (Figura $2 \mathrm{D}$ ); porém, foi significativa a parcela do solo exposto, que continuou a influenciar a resposta espectral. A maior correlação verificada pelo índice normalizado NDVI 5:7, o qual utilizou bandas do infravermelho médio, pode ser explicada pelas relações inversas (antagônicas) esperadas nas regiões do visível e do infravermelho médio, conforme Ponzoni $\&$ Resende (2004). De acordo com Lu et al. (2005) e Freitas et al. (2005), o índice (RVI 5:4), gerado a partir de banda do infravermelho médio (banda 5), apresenta potencial para a análise do vigor das plantas. Esse índice apresentou a segunda maior correlação, apesar da baixa densidade da cobertura vegetal característica do SAF 2.

$\mathrm{Na}$ classe SAF 3, as correlações observadas foram relativamente elevadas $\left(\mathrm{R}^{2}=0,78\right)$ para o índice TSAVI 2 (complexo). Os índices normalizados apresentaram a segunda maior correlação $\left(\mathrm{R}^{2}=0,66\right)$ no NDVI $4: 3$ e no NRVI ("normalized ratio vegetation index"). Ambas as correlações foram significativas a $1 \%$ de probabilidade. Uma das áreas amostrais do SAF 3, representada pela imagem do TSAVI 2 (Figura 2 E), apresentou valores com maior densidade de cobertura vegetal, característica dessa classe agroflorestal mais antiga, com recobrimento de copa superior a $60 \%$, indivíduos acima de $6 \mathrm{~m}$ de altura e $42 \mathrm{Mg} \mathrm{ha}^{-1} \mathrm{de}$ estoque de carbono.

A cobertura vegetal da área em destaque na imagem síntese (Figura $2 \mathrm{~F}$ ) indica a presença de indivíduos arbóreos já em fase avançada de crescimento, com até 12 anos. A divisão de dois estratos arbóreos e a exposição de solo no interior da vegetação também são bem caracterizadas nessa classse. Porém, o índice TSAVI 2 (complexo), utilizado por Baret \& Guyot (1991), e o NDVI (normalizado), recomendado por Huete et al. (2002), apresentaram as maiores correlações. Portanto, apesar do recobrimento de copa 

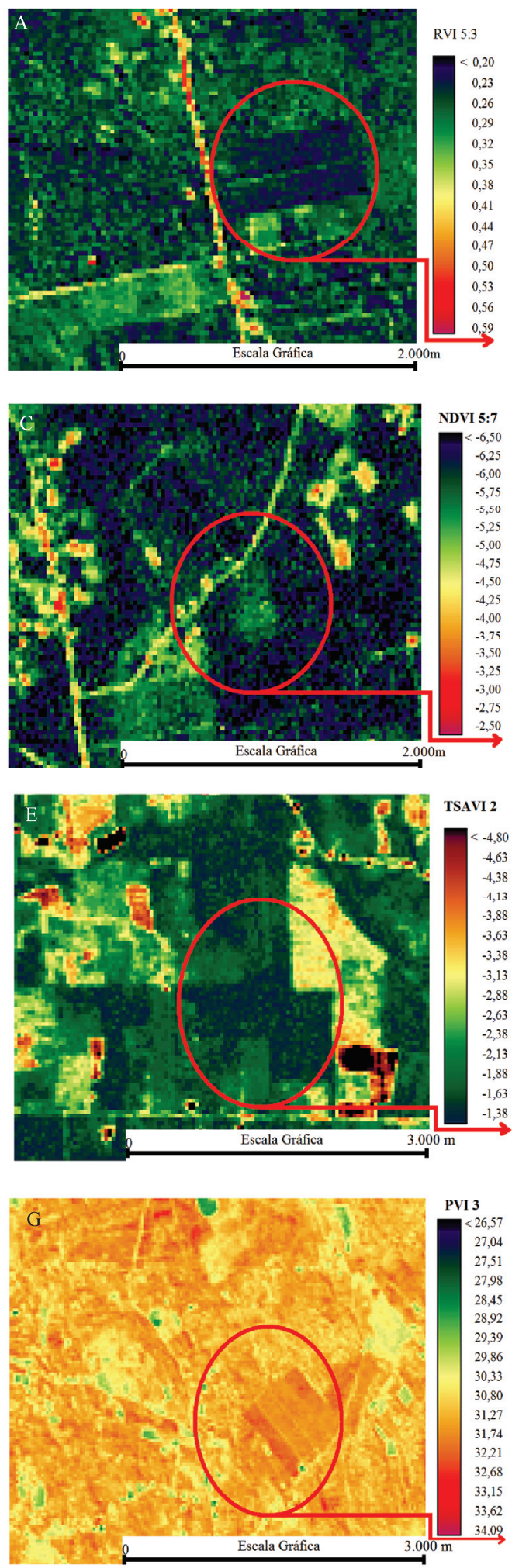

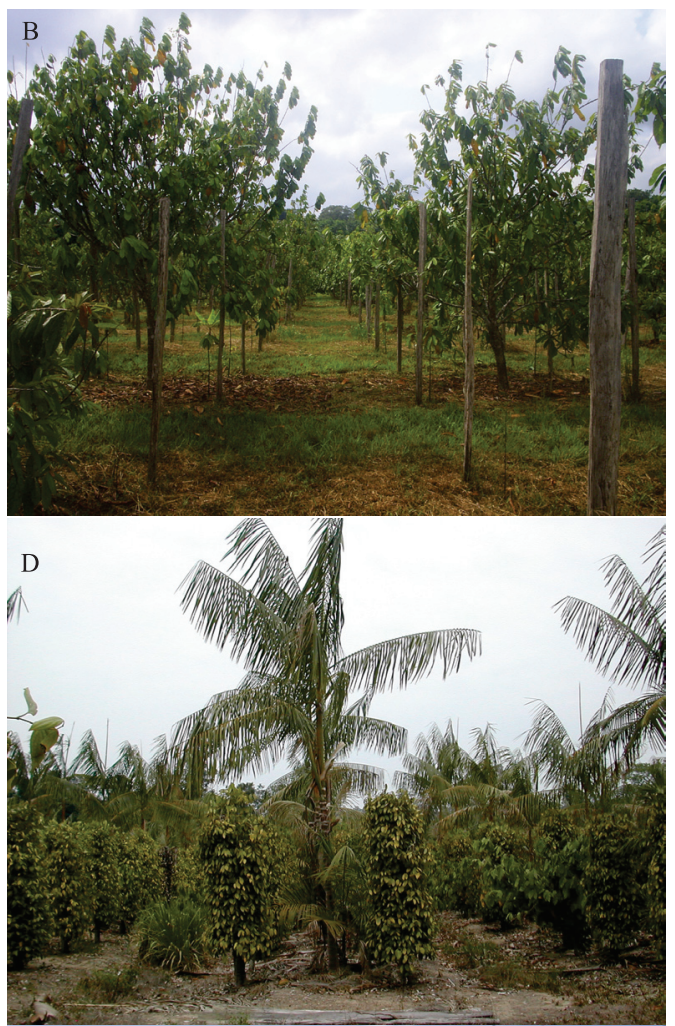

F
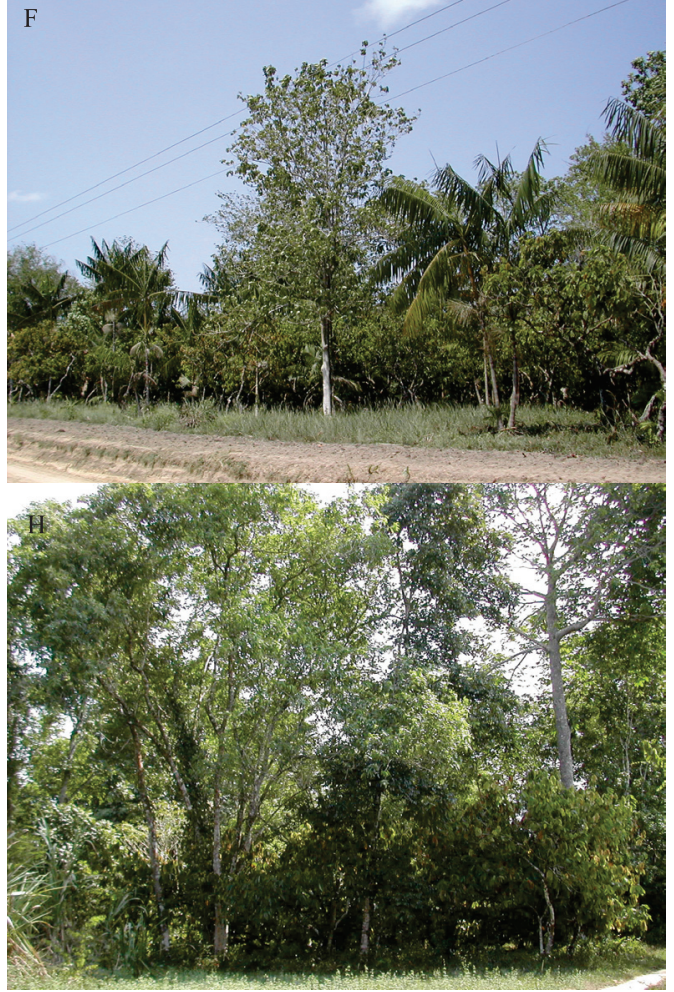

Figura 2. Imagens síntese dos sistemas agroflorestais avaliados: A, SAF 1 (RVI 5:3); B, SAF 1 em campo; C, SAF 2 (NDVI 5:7); D, SAF 2 em campo; E, SAF 3 (TSAVI 2); F, SAF 3 em campo; G, SAF 4 (PVI 3); e H, SAF 4 em campo. 
relativamente elevado nessa classe, o solo exposto também pode ter influenciado a resposta espectral, uma vez que o primeiro índice minimiza os efeitos do brilho de fundo do solo, e o segundo é recomendado para vegetações mais esparsas.

Para o SAF 4, os melhores resultados foram obtidos com os índices complexos, com valores de $\mathrm{R}^{2}$ de 0,61 para o PVI 3 e de 0,54 para o PVI 2, significativos a $1 \%$ de probabilidade. A área amostral do SAF 4, exemplificada na imagem síntese do PVI 3 (Figura $2 \mathrm{G}$ ), apresenta valores com elevada cobertura vegetal. Conforme dados de campo, esta classe mais antiga tem cobertura de copa média de $80 \%$, com indivíduos de altura superior a $7 \mathrm{~m}$ e com média de estoque de carbono de $120 \mathrm{Mg} \mathrm{ha}^{-1}$.

Indivíduos arbóreos em fase avançada de desenvolvimento (idade acima de 15 anos), com estratos arbóreos bem definidos e com baixa exposição de solo, caracterizam a estrutura vegetal da área destacada na imagem síntese representada na Figura 2 H. Embora haja influência de eventuais ruídos da atmosfera, ao se realizar operações como a multiplicação, as melhores correlações verificadas nos índices complexos - em especial nos PVIs - são explicadas pelo uso da razão de bandas e dos parâmetros de ajuste para minimizar o efeito de solo na resposta espectral da imagem, mesmo que a presença deste fosse pequena. Tanto o PVI 2 quanto o PVI 3 foram desenvolvidos para melhorar o ajuste da influência da água da vegetação, em comparação ao PVI original (Perry \& Lautenschlager, 1984). De modo geral, os índices de razão simples obtiveram correlações elevadas para a classe SAF 1 (Tabela 1), apesar das interferências atmosféricas que afetam seu valor absoluto (Liu \& Kogan, 2002). A distribuição angular das folhas, a rugosidade do dossel e outros modelos de correção atmosférica foram utilizados para minimizar a forte influência atmosférica da Região Amazônica e melhorar correlações obtidas (Lu et al., 2002).

Os índices normalizados, amplamente citados como índices com altas correlações com fatores vegetativos agrícolas ou florestais (Ponzoni \& Resende, 2004), também apresentaram correlações elevadas $\left(\mathrm{R}^{2}=0,64\right.$ a 0,73$)$, no SAF 2 (Tabela 2). A diminuição da correlação destes índices da classe SAF 2 (menor biomassa) para a SAF 4 (maior biomassa) - que variou de $\mathrm{R}^{2}=0,07$ a 0,27 - é resultante do aumento desta em condições de baixa biomassa. Isso indica que, quando se elevam os índices de área foliar, há saturação dos valores (Huete, 2002).
Em relação aos índices denominados complexos, destaca-se o TSAVI 2, com a maior correlação para a classe SAF 3, e o PVI 3, com a maior correlação para a classe SAF 4. Estes bons resultados dos índices complexos, nos sistemas agroflorestais com maior biomassa, pode resultar da aplicação dos dados obtidos pela "linha do solo" (intercepto e coeficiente angular). Segundo Ponzoni \& Resende (2004), estes índices se fundamentam no contraste espectral existente entre vegetação e características do solo, por meio da alteração na ortogonalidade dos eixos de dispersão dos dados, e são indicados para correlações com vegetação de maior biomassa.

\section{Conclusões}

1. As respostas espectrais dos sistemas agroflorestais, obtidos por meio de imagens $\mathrm{TM} /$ Landsat-5, não seguem os padrões típicos da vegetação verde e são alteradas conforme as variações de biomassa nas diferentes classes analisadas.

2. Os índices de vegetação de razão simples e de diferença normalizada são mais eficientes na predição de biomassa aérea de sistemas agroflorestais jovens, enquanto os índices complexos são mais eficientes na predição de biomassa de sistemas agroflorestais antigos.

\section{Referências}

ACCIOLY, L.J. de O.; PACHÊCO, A.; COSTA, T.C. e C.; LOPES, O.F.; OLIVEIRA, M.A.J. de. Relações empíricas entre a estrutura da vegetação e dados do sensor TM/LANDSAT. Revista Brasileira de Engenharia Agrícola e Ambiental, v.6, p.492-498, 2002.

BARET, F.; GUYOT, G. Potentials and limits of vegetation indices for LAI and APAR assessment. Remote Sensing of Environment, v.3, p.161-73, 1991.

BARTELT, D.; KOCH, J.; TOURINHO, M.M. Anbau von Acai und Kakao in Primärwäldern der Varzeas am Rio Tocantins (Brasilien/ Para). Forstarchiv, v.71, p.250-256, 2000.

BOLFE, É.L.; BATISTELLA, M. Análise florística e estrutural de sistemas silviagrícolas em Tomé-Açu, Pará. Pesquisa Agropecuária Brasileira, v.46, p.1139-1147, 2011.

BOLFE, E.L.; FERREIRA, M.C.; BATISTELLA, M. Biomassa epígea e estoque de carbono de agroflorestas em Tomé-Açu, PA. Revista Brasileira de Agroecologia, v.4, p.2171-2175, 2009.

CLEVERS, J.G.P.W. The derivation of a simplified reflectance model for the estimation of leaf area index. Remote Sensing of Environment, v.25, p.53-69, 1988.

DEMATTÊ, J.A.M.; NANNI, M.R. Weathering sequence of soils developed from basalt as evaluated by laboratory (IRIS), airborne 
(AVIRIS) and orbital (TM) sensors. International Journal Remote Sensing, v.24, p.4715-4738, 2003.

FODDY, G.M.; BOYD, D.S.; CLUTER, M.E.J. Predictive relations of tropical forest biomass from Landsat TM data and their transferability between regions. Remote Sensing of Environment, v.85, p.463-474, 2003.

FREITAS, S.R.; MELLO, M.C.S.; CRUZ, C.B.M. Relationships between forest structure and vegetation indices in Atlantic Rainforest. Forest Ecology and Management, v.218, p.353-362, 2005.

HIGUCHI, N.; SANTOS, J. dos; RIBEIRO, R.J.; MINETTE, L.; BIOT, Y. Biomassa da parte aérea da vegetação de floresta tropical úmida de terra-firme da Amazônia Brasileira. Acta Amazonica, v.28, p.153-166, 1998.

HUETE, A.; DIDAN, K.; MIURA, T.; RODRIGUEZ, E.P.; GAO, X.; FERREIRA, L.G. Overview of the radiometric and biophysical performance of the MODIS vegetation indices. Remote Sensing of Environment, v.83, p.195-213, 2002.

HUETE, A.R. A soil-adjusted vegetation index (SAVI). Remote Sensing of Environment, v.25, p.295-309, 1988.

INSTITUTO NACIONAL DE PESQUISAS ESPACIAIS. Monitoramento da floresta amazônica brasileira por satélite. 2008. Disponível em: <www.dpi.inpe.br/prodesdigital >. Acesso em: 15 ago. 2011.

JORDAN, C.F. Derivation of leaf-area index from quality of light on the forest floor. Ecology, v.50, p.663-666, 1969.

LIU, W.T.; KOGAN, F. Monitoring Brazilian soybean production using NOAA/AVHRR based vegetation condition indices. International Journal of Remote Sensing, v.23, p.1161-1179, 2002.

LU, D.; MAUSEL, P.; BRONDIZIO, E.; MORAN, E. Assessment of atmospheric correction methods for Landsat TM data applicable to Amazon basin LBA research. International Journal of Remote Sensing, v.23, p.2651-2671, 2002.

LU, D.S.; BATISTELLA, M.; MORAN, E. Satellite estimation of aboveground biomass and impacts of forest stand structure. Photogrammetric Engineering and Remote Sensing, v.71, p.967-974, 2005.

MONTAGNINI, F.; NAIR, P.K.R. Carbon sequestration: an underexploited environmental benefit of agroforestry systems. Agroforestry Systems, v.61, p.281-295, 2004.

NATIONALAERONAUTICS AND SPACE ADMINISTRATION. The Landsat Program. 2008. Available at: <http://landsat.gsfc. nasa.gov/>. Accessed on: 25 Jan. 2009.
NOBRE, C.A.; SAMPAIO, G.; SALAZAR, L. Mudanças climáticas e Amazônia. Ciência e Cultura, v.59, p.22-27, 2007.

PACHÊCO, N.A.; BASTOS, T.X. Boletim agrometeorológico 2006: Tomé-Açu, PA. Belém: Embrapa Amazônia Oriental, 2008. 30p. (Embrapa Amazônia Oriental. Documentos, 320).

PERRY, C.R.; LAUTENSCHLAGER, L.F. Functional equivalence of spectral vegetation indices. Remote Sensing of Environment, v.14, p.169-182, 1984.

PINHO, R.C.; MILLER, R.P.; ALFAIA, S.S. Agroforestry and the improvement of soil fertility: a view from Amazonia. Applied and Environmental Soil Science, v.2012, p.1-12, 2012. Doi:10.1155/2012/616383.

PONZONI, F.J.; REZENDE, A.C.P. Caracterização espectral de estágios sucessionais de vegetação secundária arbórea em Altamira (PA), através de dados orbitais. Revista Árvore, v.28, p.535-545, 2004.

QI, J.; CHEHBOUNI, A.; HUETE A.R.; KERR, Y.H.; SOROOSHIAN, S. A modified soil adjusted vegetation index. Remote Sensing of Environment, v.48, p.119-126, 1994.

RICHARDSON, A.J.; WIEGAND, C.L. Distinguishing vegetation from soil background information. Photogrammetric Engineering and Remote Sensing, v.1, p.1541-1552, 1977.

RIZZI, R.; RUDORFF, B.F.T. Imagens do sensor MODIS associadas a um modelo agronômico para estimar a produtividade de soja. Pesquisa Agropecuária Brasileira, v.42, p.73-80, 2007.

RODRIGUES, T.E.; SANTOS, P.L. dos; ROLIM, P.A.M.; SANTOS, E.; REGO, R.S.; SILVA, J.M.L. da; VALENTE, M.A.; GAMA, J.R.N.F. Caracterização e classificação dos solos do Município de Tomé-Açu, Pará. Belém: Embrapa Amazônia Oriental, 2001. 49p. (Embrapa Amazônia Oriental. Documentos, 117).

ROUSE, J.W.; HAAS, R.H.; SCHELL, J.A.; DEERING, D.W.; HARLAN, J.C. Monitoring the vernal advancement and retrogradation (greenwave effect) of natural vegetation. Greenbelt: NASA, 1974. 371p. (NASA/GSFC. Final report).

SILLEOS, N.G.; ALEXANDRIDIS, T.K.; GITAS, I.Z.; PERAKIS, $\mathrm{K}$. Vegetation indices: advances made in biomass estimation and vegetation monitoring in the last 30 years. Geocarto International, v.21, p.21-28, 2006.

TSUKAMOTO, A. de A.; COUTO, L.; NEVES, J.C.L.; PASSOS, C.A.M.; SILVA, M.L. Fixação de carbono em um sistema agrissilvipastoril com eucalipto na região do cerrado de Minas Gerais. Agrossilvicultura, v.1, p.29-41, 2004.

$\overline{\text { Recebido em } 11 \text { de setembro de } 2011 \text { e aprovado em } 20 \text { de agosto de } 2012}$ 